\title{
TOPOLOGICAL COMPLEXITY OF WEDGES AND COVERING MAPS
}

\author{
ALEXANDER DRANISHNIKOV
}

(Communicated by Daniel Ruberman)

\begin{abstract}
We present some results supporting the Iwase-Sakai conjecture about coincidence of the topological complexity $\mathrm{TC}(X)$ and monoidal topological complexity $\mathrm{TC}^{M}(X)$. Using these results we provide lower and upper bounds for the topological complexity of the wedge $X \vee Y$. We use these bounds to give a counterexample to the conjecture asserting that $\mathrm{TC}\left(X^{\prime}\right) \leq \mathrm{TC}(X)$ for any covering map $p: X^{\prime} \rightarrow X$.

Also we discuss a possible reduction of the monoidal topological complexity to the Lusternik-Schnirelmann category.
\end{abstract}

\section{INTRODUCTION}

Let $P X=X^{[0,1]}$ denote the space of all paths in a path connected space $X$. Let $i_{X}: X \rightarrow P X$ be the inclusion of $X$ into $P X$ as a subspace of constant paths. There is a natural fibration $\pi: P X \rightarrow X \times X$ defined as $\pi(f)=(f(0), f(1))$ for $f \in P X, f:[0,1] \rightarrow X$.

Let $X$ be an ENR. A piecewise continuous section $s: X \times X \rightarrow P X$ of $\pi$ is called a motion planning algorithm. We say that a motion planning algorithm $s$ has complexity $k$ if $X \times X$ can be presented as a disjoint union $F_{0} \cup \cdots \cup F_{k}$ of $k+1$ ENRs such that $s$ is continuous on each $F_{i}$. The normalized topological complexity $\mathrm{TC}(X)$ of a space $X$ was defined by Farber as the minimum $k$ such that there is a motion planning algorithm of complexity $k[\mathrm{~F} 1$. Equivalently, $\mathrm{TC}(X)$ is the minimal number $k$ such that $X \times X$ admits an open cover $U_{0}, \ldots, U_{k}$ by $k+1$ sets such that over each $U_{i}$ there is a continuous section of $\pi$.

We say that a motion planning algorithm $s: X \times X \rightarrow P X$ is reserved if $\left.s\right|_{\Delta X}=i_{X}$, where $\Delta X \subset X \times X$ is the diagonal. In other words, if the initial position of a robot in the configuration space $X$ coincides with the terminal position, then the algorithm keeps the robot still. This condition on the motion planning algorithms seems to be very natural. The corresponding complexity of a space $X$ was denoted by Iwase and Sakai as $\mathrm{TC}^{M}(X)$ and was called the monoidal topological complexity of $X$ [IS1]. In the original definition they additionally assumed that all sets $U_{i}$ contain the diagonal. Their definition agrees with the above since their condition can always be achieved by reduction of an open cover $U_{0}, \ldots, U_{k}$ with reserved sections $s_{i}$ to a closed cover $F_{0}, \ldots, F_{k}, F_{i} \subset U_{i}$, then by adding the

Received by the editors July 31, 2012 and, in revised form, August 11, 2012; August 12, 2012; September 25, 2012; and January 15, 2013.

2010 Mathematics Subject Classification. Primary 55M30; Secondary 57N65, 54F45.

This work was supported by NSF grant DMS-0904278. 
diagonal to each $F_{i}$ with the natural extension of the sections $\bar{s}_{i}$, and then by taking an open enlargement $V_{i}$ of the sets $F_{i} \cup \Delta X$ that admit extensions of the sections $\bar{s}_{i}$.

Iwase and Saki conjectured that $\operatorname{TC}^{M}(X)=\mathrm{TC}(X)$. In fact, first they gave a proof to the conjecture in IS1 and then withdrew it in IS2]. We prove this conjecture under the assumption that $\mathrm{TC}(X)>\operatorname{dim} X$. Also, using the Weinberger Lemma from [F3] we show that the conjecture holds true when $X$ is a Lie group.

The topological complexity is closely related to the Lusternik-Schnirelmann category $\operatorname{cat}_{\mathrm{LS}}(X)$ of a space which is defined as the minimal number $k$ such that $X$ can be covered by $k+1$ open sets $U_{0}, U_{1}, \ldots, U_{k}$, all contractible to a point in $X$. Throughout the paper we use the normalized LS-category (as well as the normalized topological complexity), since they improve several important inequalities in the theory:

$$
\operatorname{cup} \text {-length }(X) \leq \operatorname{cat}_{\mathrm{LS}}(X) \leq \operatorname{dim}(X) \text { and } \operatorname{cat}_{\mathrm{LS}}(X \times Y) \leq \operatorname{cat}_{\mathrm{LS}}(X)+\operatorname{cat}_{\mathrm{LS}}(Y) \text {, }
$$

where in the first inequality the cup-length is taken for any reduced cohomology (possibly twisted) CLOT.

Some of the formulas for cat $_{L S}$ translate to similar statements for TC. For example, for TC there is an inequality similar to the above for the product of two spaces [F4. Also, there are analogous estimates of TC in terms of the cup product and dimension [F4. On the other hand, the simple cat ${ }_{L S}$ formula for the wedge $\operatorname{cat}_{\mathrm{LS}}(X \vee Y)=\max \left\{\operatorname{cat}_{\mathrm{LS}} X, \operatorname{cat}_{\mathrm{LS}} Y\right\}$ does not hold for TC. So far there is no good analog of it for TC. The best that we can prove here is Theorem 3.6 from this paper. Another example is the formula $\operatorname{cat}_{\mathrm{LS}}(Y) \geq \operatorname{cat}_{\mathrm{LS}}(X)$ for a covering map $p: X \rightarrow Y$ which supports an intuitive idea that a covering space is always simpler than the base. So it was natural to assume that the same holds true for TC. We learned about this problem from Yuli Rudyak. In this paper Theorem 3.8 gives a negative answer to this question.

There have been several attempts to reformulate the topological complexity of $X$ as some modified category of a related space. Thus, Garcia Calcines and Vandembroucq defined the weak topological complexity wTC $(X)$ GV1] in the spirit of Berstein-Hilton's $[\mathrm{BH}]$ definition of the weak category wcat $(X)$. They proved the equality wTC $(X)=\operatorname{wcat}(X \times X) / \Delta X$ and found some sufficient conditions for the equality $\mathrm{wTC}(X)=\mathrm{TC}(X)$. Then in GV2 they established the equality $\mathrm{TC}(X)=\operatorname{cat}_{\mathrm{LS}}(X \times X) / \Delta X$ for large classes of spaces. In this paper we discuss a possibility of such equality for the monoidal topological complexity: $\mathrm{TC}^{M}(X)=\operatorname{cat}_{\mathrm{LS}}(X \times X) / \Delta X$. We define a rel $\infty$ category $\infty$ - $\operatorname{cat}_{\mathrm{LS}}(Y)$ of noncompact spaces $Y$ and discuss the problem of coincidence between $\operatorname{cat}_{\mathrm{LS}}(X / A)$ and $\infty$ - $\operatorname{cat}_{\mathrm{LS}}(X \backslash A)$ for a subcomplex $A \subset X$ of a finite complex $X$. Then we show that $\mathrm{TC}^{M}(X)$ is bounded between $\operatorname{cat}_{\mathrm{LS}}(X \times X) / \Delta X$ and $\infty$ - $\operatorname{cat}_{\mathrm{LS}}(X \times X \backslash \Delta X)$.

Note that for the topological complexity Farber proved the inequality [F2]

$$
\operatorname{cat}_{\mathrm{LS}}(X \times X) / \Delta X-1 \leq \mathrm{TC}(X) .
$$

Thus, in view of the Iwase-Sakai conjecture, examples of spaces for which Farber's inequality is sharp are of great interest.

We recall that both $\operatorname{cat}_{\mathrm{LS}}(X)$ and $\mathrm{TC}(X)$ are special cases of the Schwarz genus [Sch]: $\operatorname{cat}_{\mathrm{LS}}(X)=s g\left(\pi_{0}: P_{0} X \rightarrow X\right)$ and $\mathrm{TC}(X)=s g(\pi: P X \rightarrow X \times X)$, where $P_{0} X \subset P X$ is the subspace of paths $f:[0,1] \rightarrow X$ that start in a base point $x_{0} \in X, f(0)=x_{0}$, and $\pi_{0}(f)=f(1)$. We recall that the normalized Schwarz 
genus [Sch] (also known as the sectional category [J]) of a fibration $p: X \rightarrow Y$ is the minimal number of open sets $U_{0}, \ldots, U_{k}$ that cover $Y$ and admit sections $s_{i}: U_{i} \rightarrow X$ of $p$. In this paper we estimate the Schwarz genus [Sch] of arbitrary fibration $p: X \rightarrow Y$ in terms of the category of its mapping cone $C_{p}$,

$$
\operatorname{cat}_{\mathrm{LS}}\left(C_{p}\right)-1 \leq s g(p) \leq \infty-\operatorname{cat}_{\mathrm{LS}}\left(C_{p} \backslash\{*\}\right) .
$$

Similar estimates for the weak sectional category in terms of wcat $\left(C_{p}\right)$ were made in GV1]:

$$
\operatorname{wcat}\left(C_{p}\right)-1 \leq w \operatorname{secat}(p) \leq \operatorname{wcat}\left(C_{p}\right) .
$$

The similarity would be complete if the problem about the equality $\operatorname{cat}_{\mathrm{LS}} X / A=$ $\infty$ - $\operatorname{cat}_{\mathrm{LS}}(X \backslash A)$ could be solved affirmatively.

\section{Monoidal topological COMplexity}

Theorem 2.1. For ENR spaces,

$$
\mathrm{TC}(X) \leq \mathrm{TC}^{M}(X) \leq \mathrm{TC}(X)+1 .
$$

This theorem was proved in [IS2]. Since the proof there is too technical, we give an alternative proof.

Proof. The first inequality is obvious. Since $X$ is ANR, there is an open neighborhood $W$ of the diagonal $\Delta X$ in $X \times X$ and a continuous map $\phi: W \times[0,1] \rightarrow X$ such that $\phi\left(x, x^{\prime}, 0\right)=x, \phi\left(x, x^{\prime}, 1\right)=x^{\prime}$, and $\phi(x, x, t)=x$ for all $t \in[0,1],\left(x, x^{\prime}\right) \in W$. Let $\mathrm{TC}(X)=n$ and let $U_{0}, U_{1}, \ldots, U_{n}$ be an open cover of $X \times X$ by sets that admit sections $s_{i}: U_{i} \rightarrow P X$ of $\pi$. Let $F$ be a closed neighborhood of $\Delta X$ that lies in $W$. Then all sets in the open cover $U_{0} \backslash F, \ldots, U_{n} \backslash F, W$ of $X \times X$ admit reserved sections. Hence $\mathrm{TC}^{M}(X) \leq n+1$.

Note that the path fibration $\pi: P X \rightarrow X \times X$ restricted over the diagonal defines the free loop fibration $p: L X \rightarrow X$. A canonical section $\bar{s}: \Delta X \rightarrow L X$ of $p$ is defined as $\bar{s}(x)=c_{x}$, where $c_{x}: I \rightarrow X$ is the constant map to $x$.

We use the standard convention to denote the elements of the iterated join product $X_{0} * \cdots * X_{n}$ as formal linear combinations $t_{0} x_{0}+t_{1} x_{1}+\cdots+t_{n} x_{n}, \sum t_{i}=1$, $t_{i} \geq 0, x_{i} \in X_{i}$, where all summands of the type $0 x_{i}$ are dropped. We use the notation $*^{n} X$ for the iterated join product of $n$ copies of $X$ with itself.

We recall that a fiberwise join of maps $f_{i}: X_{i} \rightarrow Y, i=0, \ldots, n$, is the map

$$
f_{0} \tilde{*} \cdots \tilde{*} f_{n}: X_{0} \tilde{*}_{Y} \cdots \tilde{*}_{Y} X_{n} \rightarrow Y
$$

where

$$
X_{0} \tilde{*}_{Y} \cdots \tilde{*}_{Y} X_{n}=\left\{t_{0} x_{0}+\cdots+t_{n} x_{n} \in X_{0} * \cdots * X_{n} \mid f_{0}\left(x_{0}\right)=\cdots=f_{n}\left(x_{n}\right)\right\}
$$

is the fiberwise join of spaces $X_{0}, \ldots, X_{n}$ and

$$
\left(f_{0} \tilde{*} \ldots \tilde{*} f_{n}\right)\left(t_{0} x_{0}+\cdots+t_{n} x_{n}\right)=f_{i}\left(x_{i}\right) .
$$

Thus, the preimage $\left(f_{0} \tilde{*} \cdots \tilde{*} f_{n}\right)^{-1}(y)$ of a point $y \in Y$ is the join product of the preimages $f_{0}^{-1}(y) * \cdots * f_{n}^{-1}(y)$.

We define $P_{n} X=P X \tilde{*}_{X \times X} \cdots \tilde{*}_{X \times X} P X$ and

$$
\pi_{n}=\pi \tilde{*} \cdots \tilde{*} \pi: P_{n} X \rightarrow X \times X
$$

to be the fiberwise join product of $n+1$ copies of $\pi$. Note that there are imbeddings $P X=P_{0} X \subset P_{1} X \subset \cdots \subset P_{n} X$ such that $\left.\pi_{i}\right|_{P_{i-1}(X)}=\pi_{i-1}$. Then the section $\bar{s}: X \times X \rightarrow P X$ of $\pi$ can be regarded as a section of $\pi_{n}$. Also we define 
$p_{0}=p: L X \rightarrow X, L_{n} X=L_{n-1} X \tilde{*}_{X} L X$, and $p_{n}=p_{n-1} \tilde{*} p: L_{n} X \rightarrow X$. Note that $\pi_{n}^{-1}(\Delta X) \cong L_{n} X$ and $p_{n}$ is the restriction of $\pi_{n}$ to $\pi_{n}^{-1}(\Delta X)$. Note also that the canonical section $\bar{s}$ defines a trivial subbundle $p_{n}^{\prime}: E \rightarrow X$ of $p_{n}$ with the fiber $n$-simplex $\Delta^{n}$.

We recall that a map $p: E \rightarrow B$ satisfies the Homotopy Lifting Property for a $\operatorname{pair}(X, A)$ if for any homotopy $H: X \times I \rightarrow B$ with a lift $H^{\prime}: A \times I \rightarrow E$ of the restriction $\left.H\right|_{A \times I}$ and a lift $H_{0}$ of $\left.H\right|_{X \times 0}$ which agrees with $H^{\prime}$, there is a lift $\bar{H}: X \times I \rightarrow E$ of $H$ which agrees with $H_{0}$ and $H^{\prime}$. We recall that a pair of spaces $(X, A)$ is called an NDR pair if $A$ is a deformation retract of a neighborhood in $X$. A pair $(X, A)$ is NDR if and only if the inclusion $A \rightarrow X$ is a closed cofibration [TD]. The following is well-known; see [TD], Corollary 5.5.3:

Theorem 2.2. Any Hurewicz fibration $p: E \rightarrow B$ satisfies the Homotopy Lifting Property for $N D R$ pairs $(X, A)$.

Corollary 2.3. Let $p: E \rightarrow X$ be a Hurewicz fibration with a section $s: X \rightarrow E$. $A$ fiberwise homotopy $G: A \times I \rightarrow E$ of the restriction $\left.s\right|_{A}$ to a closed subset $A \subset X$ can be extended to a fiberwise homotopy $\bar{G}: X \times I \rightarrow E$ of $s$ provided $(X, A)$ is an NDR pair.

Proposition 2.4. For $C W$ complexes $X$,

(1) $\mathrm{TC}(X) \leq n \Leftrightarrow \pi_{n}: P_{n} X \rightarrow X \times X$ admits a section.

(2) $\mathrm{TC}^{M}(X) \leq n \Leftrightarrow \pi_{n}: P_{n} X \rightarrow X \times X$ admits a section s which agrees with the canonical section over the diagonal, $\left.s\right|_{\Delta X}=\bar{s}$.

Proof. Statement (1) is a part of a general theorem proven by Schwarz [Sch] for fibrations $q: X \rightarrow Y, s g(q) \leq n$ if and only if the fiberwise join product $\tilde{*}^{n+1} q$ : $\tilde{*}_{Y}^{n+1} X \rightarrow Y$ of $n+1$ copies of $q$ admits a section.

The implication $\Leftarrow$ in $(2)$ is obvious. For the other direction we note that $n+1$ reserved sections $s_{i}: U_{i} \rightarrow P X$ defined for an open cover $U_{0}, \ldots, U_{n}$ of $X \times X$ define a section $s$ of $\pi_{n}$ with the image $s(\Delta X)$ lying in $E$. Therefore over $\Delta X$ it could be fiberwise deformed to $\bar{s}$. By Theorem 2.2 that deformation can be extended to a fiberwise deformation of $s$.

Theorem 2.5. The equality

$$
\mathrm{TC}(X)=\mathrm{TC}^{M}(X)
$$

holds true for $k$-connected simplicial complexes $X$ such that

$$
(k+1)(\mathrm{TC}(X)+1)>\operatorname{dim} X+1 .
$$

Proof. Let $\mathrm{TC}(X)=n$. Note that the fiber $\pi^{-1}\left(x, x^{\prime}\right)$ is homotopy equivalent to the loop space $\Omega(X)$. Since $\Omega(X)$ is $(k-1)$-connected, the iterated join product $*^{n+1} \Omega(X)$ is $((k+1)(n+1)-2)$-connected. We show that any section $s: \Delta X \rightarrow$ $L_{n} X$ can be joined by a fiberwise homotopy to the canonical section $\bar{s}: \Delta X \rightarrow$ $L_{n} X$. By induction on $i$ we construct a section $s_{i}: X \rightarrow L_{n} X$, that coincides with $\bar{s}$ on the $i$-skeleton $X^{(i)}$, together with a fiberwise homotopy joining $s$ and $s_{i}$. Here we use the identification $\Delta X=X$. For $i=0$ we take paths in the fibers $p_{n}^{-1}(v)$ joining $s(v)$ and $\bar{s}(v)$ for all $v \in X^{(0)}$. Then we extend them to a fiberwise homotopy of $s$ to a section $s_{0}$. Assume that $s_{i-1}$ is already constructed and $i \leq \operatorname{dim} X \leq(k+1)(n+1)-2$. Independently for every $i$-simplex $\sigma \subset X$ we consider the problem of joining $s_{i-1}$ with $\bar{s}$ over $\sigma$ by a fiberwise homotopy. Since 
the fiber bundle $p_{n}$ is trivial over $\sigma$ with an $i$-connected fiber, the identity homotopy on the boundary $\partial \sigma$ can be extended to a homotopy between $\left.\bar{s}\right|_{\sigma}$ and $\left.s_{i-1}\right|_{\sigma}$. This extension can be deformed to a fiberwise homotopy. All these homotopies together define a fiberwise homotopy between $s_{i-1}$ and $\bar{s}$ over $X^{(i)}$. Since $\left(X, X^{(i)}\right)$ is an NDR pair, by Corollary 2.3 we can extend it to a fiberwise homotopy over $X$.

Let $s: X \times X \rightarrow P_{n} X$ be a section. We proved that on $\Delta X$ it can be deformed to a canonical section $\bar{s}$. Since $(X \times X, \Delta X)$ is an NDR pair, by Corollary 2.3 there is a fiberwise homotopy of $s$ to a section $s^{\prime}$ that coincides with $\bar{s}$ on $\Delta X$. Therefore, $\mathrm{TC}^{M}(X) \leq n$.

Corollary 2.6. $\mathrm{TC}\left(S^{n}\right)=\mathrm{TC}^{M}\left(S^{n}\right)$ for $n>1$.

The case of $n=1$ is taken care of by Lemma 2.7.

The following is an extension of Weinberger's Lemma from [F3 to the case of monoidal topological complexity.

Lemma 2.7. For a connected Lie group $G$,

$$
\mathrm{TC}(G)=\mathrm{TC}^{M}(G)=\operatorname{cat}_{\mathrm{LS}}(G) .
$$

Proof. In view of what is already known [F3, it suffices to show the inequality $\mathrm{TC}^{M}(G) \leq \operatorname{cat}_{\mathrm{LS}}(G)$. Let $\operatorname{cat}_{\mathrm{LS}}(G)=n$ and let $U_{0}, U_{1}, \ldots, U_{n}$ be an open cover of $G$ together with homotopies $H_{i}: U_{i} \times[0,1] \rightarrow G$ contracting $U_{i}$ to the unit $e \in G$. Clearly, we may assume that $e \notin U_{i}$ for $i>0$. Since the inclusion $e \in G$ is a cofibration, we may assume that $H_{0}(e, t)=e$ for all $t$ (see Lemma 1.25 of [CLOT]). Then for the open cover of $G \times G$ as defined in [F3],

$$
W_{i}=\left\{(a, b) \in G \times G \mid b^{-1} a \in U_{i}\right\},
$$

the sections $s_{i}: W_{i} \rightarrow P G$ defined by the formula

$$
s_{i}(a, b)(t)=b H_{i}\left(b^{-1} a, t\right) \in G, \quad(a, b) \in W_{i},
$$

are reserved. Indeed, $\Delta G \cap W_{i}=\varnothing$ for $i>0$ and

$$
s_{0}(a, a)(t)=a H_{0}\left(a^{-1} a, t\right)=a H_{0}(e, t)=a e=a
$$

for all $(a, a) \in \Delta G$.

\section{TOPOLOGICAL COMPLEXITY OF WEDGE AND COVERING MAPS}

A deformation of $U \subset Z$ in $Z$ to a subset $A \subset Z$ is a continuous map $D: U \times I \rightarrow$ $Z$ such that $D(u, 0)=u, D(u, 1) \in A$ for all $u \in U$. A strict deformation of $U \subset Z$ in $Z$ to $A \subset Z$ is a deformation $D: U \times I \rightarrow Z$ such that $D(u, t)=u$ for all $t \in I$ whenever $u \in A$.

Proposition 3.1. Let $X$ be a metric space. For an open set $U \subset X \times X$ the following are equivalent:

(1) There is a reserved section $s: U \rightarrow P X$ over $U$ of the fibration $\pi: P X \rightarrow$ $X \times X$.

(2) There is a strict deformation $D: U \times I \rightarrow X \times X$ to the diagonal $\Delta X=$ $\{(x, x) \in X \times X \mid x \in X\}$.

(3) For any choice of a base point $x_{0} \in X$ there is a strict deformation $D$ of $U$ to $\Delta X$ which preserves faces $X \times x_{0}$ and $x_{0} \times X$; i.e., for all $t \in I$,

$$
D\left(\left(x, x_{0}\right), t\right) \in X \times x_{0} \quad \text { and } \quad D\left(\left(x_{0}, x\right), t\right) \in x_{0} \times X .
$$


Proof. $(1) \Rightarrow(3)$. Let $\|x\|=d\left(x, x_{0}\right)$. We define

$$
D((x, y), t)=s(x, y)\left(\frac{\|x\|}{\|x\|+\|y\|} t\right), s(x, y)\left(1-\frac{\|y\|}{\|x\|+\|y\|} t\right)
$$

if $(x, y) \neq\left(x_{0}, x_{0}\right)$ and define $D\left(\left(x_{0}, x_{0}\right), t\right)=\left(x_{0}, x_{0}\right)$. Since $s(x, y)(0)=x$ and $s(x, y)(1)=y$, we obtain that $D((x, y), 0)=(x, y)$. Note that

$$
D((x, y), 1)=s(x, y)\left(\frac{\|x\|}{\|x\|+\|y\|}\right), s(x, y)\left(\frac{\|x\|}{\|x\|+\|y\|}\right) \in \Delta X .
$$

Since the section $s$ is reserved, $D((x, x), t)=(s(x, x)(t / 2), s(x, x)(t / 2))=(x, x)$. Note that

$$
D\left(\left(x, x_{0}\right), t\right)=\left(s\left(x, x_{0}\right)(t), s\left(x, x_{0}\right)(1)\right)=\left(s\left(x, x_{0}\right)(t), x_{0}\right) \in X \times x_{0}
$$

and

$$
D\left(\left(x_{0}, y\right), t\right)=\left(s\left(x_{0}, y\right)(0), s\left(x_{0}, y\right)(1-t)\right)=\left(x_{0}, s\left(x_{0}, y\right)(1-t)\right) \in x_{0} \times X .
$$

The deformation $D$ is continuous at $\left(x_{0}, x_{0}\right)$ since the section $s\left(x_{0}, x_{0}\right)$ is stationary at $\left(x_{0}, x_{0}\right)$.

$(3) \Rightarrow(2)$ is obvious.

$(2) \Rightarrow(1)$. Let $p r_{1}: X \times X \rightarrow X$ denote the projection to the first factor and $p r_{2}: X \times X \rightarrow X$ to the second. Given a strict deformation $D: U \times I \rightarrow X \times X$ we define a section $s: U \rightarrow P X$ as follows:

$$
s(x, y)(t)= \begin{cases}p r_{1} D((x, y), 2 t) & \text { if } t \leq 1 / 2 \\ \operatorname{pr}_{2} D((x, y), 2-2 t) & \text { if } t \geq 1 / 2\end{cases}
$$

This path is well-defined since $D((x, y), 1) \in \Delta X$. Clearly it is a path from $x$ to $y$. If $x=y$, the path is stationary. Thus $s$ is a reserved section.

The following lemma was proven in [F4, Lemma 4.25.

Lemma 3.2. Let $A$ be a retract of an ENR space $X$. Then $\operatorname{TC}(X) \geq \operatorname{TC}(A)$.

We recall that a family $\mathcal{U}$ of subsets of $X$ is called a $k$-cover, $k \in \mathbb{N}$, if every subfamily of $\mathcal{U}$ that consists of $k$ elements forms a cover of $X$. We use the following theorem $[\mathrm{Dr}$.

Theorem 3.3. Let $\left\{U_{0}^{\prime}, \ldots, U_{n}^{\prime}\right\}$ be an open cover of a normal topological space $X$. Then for any $m=n, n+1, \ldots, \infty$ there is an open $(n+1)$-cover of $X,\left\{U_{k}\right\}_{k=0}^{m}$ such that $U_{k}=U_{k}^{\prime}$ for $k \leq n$ and $U_{k}=\bigcup_{i=0}^{n} V_{i}$ is a disjoint union with $V_{i} \subset U_{i}$ for $k>n$.

Corollary 3.4. Suppose that all sets $U_{i}^{\prime}, i=0, \ldots, n$, in the theorem are (strictly) deformable in $X$ to a subspace $A \subset X$. Then the sets $U_{k}$ for all $k$ are (strictly) deformable in $X$ to $A$.

The following proposition is well-known. It can be traced back to the work of Kolmogorov on Hilbert's 13th problem Os.

Proposition 3.5. Let $\mathcal{U}=\left\{U_{0}, \ldots, U_{n+m}\right\}$ be an $(n+1)$-cover of $X$ and let $\mathcal{V}=\left\{V_{0}, \ldots, V_{m+n}\right\}$ be an $(m+1)$-cover of $Y$. Then the sets $W_{k}=U_{k} \times V_{k}$, $k=0, \ldots, n+m$, cover $X \times Y$. 
Proof. Let $(x, y) \in X \times Y$. A point $x$ is covered by at least $m+1$ elements of $\mathcal{U}$. Otherwise $n+1$ elements that do not cover $x$ would not form a cover of the space $X$. That would give a contradiction with the assumption that $\mathcal{U}$ is an $(n+1)$-cover of $X$. Let $x \in U_{i_{0}} \cap \cdots \cap U_{i_{m}}$. By the assumption, the family $V_{i_{0}}, \ldots, V_{i_{m}}$ covers $Y$. Hence $y \in V_{i_{s}}$ for some $s$. Then $(x, y) \in W_{i_{s}}$.

Theorem 3.6. For all ANR spaces $X$ and $Y$,

$$
\begin{gathered}
\max \left\{\mathrm{TC}(X), \mathrm{TC}(Y), \operatorname{cat}_{\mathrm{LS}}(X \times Y)\right\} \leq \mathrm{TC}(X \vee Y) \\
\leq \mathrm{TC}^{M}(X \vee Y) \leq \mathrm{TC}^{M}(X)+\mathrm{TC}^{M}(Y) .
\end{gathered}
$$

Proof. Note that TC $(X \vee Y) \geq \mathrm{TC}(X), \mathrm{TC}(Y)$ by Lemma 3.2, Let $r_{X}: X \vee Y \rightarrow X$ and $r_{Y}: X \vee Y \rightarrow Y$ be the retractions collapsing the wedge onto $X$ and $Y$ respectively. We may assume that the subset

$$
X \times Y \subset(X \vee Y) \times(X \vee Y)
$$

is covered by $\leq \mathrm{TC}(X \vee Y)+1$ open sets $U$ supplied with homotopies

$$
H_{U}: U \times I \rightarrow X \vee Y
$$

such that $H_{U}(x, y, 0)=x$ and $H_{U}(x, y, 1)=y$. For each $U$ we define a homotopy $G_{U}: U \times I \rightarrow X \times Y$ by the formula

$$
G_{U}(x, y, t)=\left(r_{X} H_{U}(x, y, t), r_{Y} H_{U}(x, y, 1-t)\right) .
$$

Then

$$
G_{U}(x, y, 0)=\left(r_{X} H_{U}(x, y, 0), r_{Y} H_{U}(x, y, 1)\right)=\left(r_{X}(x), r_{Y}(y)\right)=(x, y)
$$

and

$$
G_{U}(x, y, 1)=\left(r_{X} H_{U}(x, y, 1), r_{Y} H_{U}(x, y, 0)\right)=\left(r_{X}(y), r_{Y}(x)\right)=\left(v_{0}, v_{0}\right),
$$

where $v_{0}$ is the wedge point in $X \vee Y$. Thus, $G_{U}$ contracts $U$ to a point in $X \times Y$.

Let $\mathrm{TC}^{M}(X)=n$ and $\mathrm{TC}^{M}(Y)=m$. Then there is an open cover $\tilde{U}_{0}, \ldots, \tilde{U}_{n}$ of $X \times X$ with reserved sections $s_{i}: \tilde{U}_{i} \rightarrow P X, i=0, \ldots, n$. Similarly, let $\tilde{V}_{0}, \ldots, \tilde{V}_{m}$ be an open covering of $Y \times Y$ with reserved sections $\sigma_{j}: \tilde{V}_{j} \rightarrow P Y$, $j=0, \ldots, m$. By Proposition 3.1 all these sets are strictly deformable to the diagonal in $X \times X$ and $Y \times Y$ respectively. By Corollary 3.4 there is an open $(n+1)$-cover $\tilde{U}_{0}, \ldots, \tilde{U}_{n}, \ldots, \tilde{U}_{n+m}$ of $X \times X$ by sets strictly deformable to the diagonal. By Proposition 3.1 there are strict deformations

$$
D_{X}^{k}: \tilde{U}_{k} \times I \rightarrow X \times X
$$

of $\tilde{U}_{k}$ to $\Delta X$ that preserve faces $X \times v_{0}$ and $v_{0} \times X$. Similarly, there is an open $(m+1)$-cover $\tilde{V}_{0}, \ldots, \tilde{V}_{m}, \ldots, \tilde{V}_{m+n}$ of $Y \times Y$ and there are strict deformations $D_{Y}^{k}$ of $\tilde{V}_{k}$ in $Y \times Y$ to the diagonal $\Delta Y$ that preserve faces.

We use the notation

$$
U_{k}=\tilde{U}_{k} \cap\left(X \times v_{0}\right) \text { and } V_{k}=\tilde{V}_{k} \cap\left(v_{0} \times Y\right), \quad k=0, \ldots, m+n .
$$

Note that $U_{0}, \ldots, U_{m+n}$ is an $(n+1)$-cover of $X \times v_{0}=X$ and $V_{0}, \ldots, V_{m+n}$ is an $(m+1)$-cover of $v_{0} \times Y=Y$. Let $W_{k}=U_{k} \times V_{k}$. By Proposition 3.5, $W_{0}, \ldots, W_{m+n}$ is an open cover of $X \times Y$. 
The deformations $D_{X}^{k}$ define the deformations $H_{k}: U_{k} \times I \rightarrow X \times v_{0}$ to the point $v_{0} \in X$ and the deformations $D_{Y}^{k}$ define the deformations $G_{k}: V_{k} \times I \rightarrow v_{0} \times Y$ to the point $v_{0} \in Y$. These deformations define the deformations

$$
T_{k}: W_{k} \times I \rightarrow X \times Y
$$

to the point $\left(v_{0}, v_{0}\right)$ such that if $W_{k} \cap\left(X \times v_{0}\right) \neq \varnothing$, then $W_{k} \cap\left(X \times v_{0}\right)=U_{k}$ and $\left.T_{k}\right|_{U_{k} \times I}=H_{k}$, and if $W_{k} \cap\left(v_{0} \times Y\right) \neq \varnothing$, then $W_{k} \cap\left(v_{0} \times Y\right)=V_{k}$ and $\left.T_{k}\right|_{V_{k} \times I}=G_{k}$ for $k=0, \ldots, m+n$.

Symmetrically, define

$$
U_{k}^{\prime}=\tilde{U}_{k} \cap\left(v_{0} \times X\right) \text { and } \quad V_{k}^{\prime}=\tilde{V}_{k} \cap\left(Y \times v_{0}\right), \quad k=0, \ldots, m+n,
$$

and the corresponding deformations

$$
H_{k}^{\prime}: U_{k}^{\prime} \times I \rightarrow X \text { and } G_{k}^{\prime}: V_{k}^{\prime} \times I \rightarrow Y
$$

to the base points. Define $W_{k}^{\prime}=U_{k}^{\prime} \times V_{k}^{\prime}$. By Proposition 3.5, the family $W_{0}^{\prime}, \ldots$, $W_{n+m}^{\prime}$ is an open cover of $Y \times X$. As before there are deformations

$$
T_{k}^{\prime}: W_{k}^{\prime} \times I \rightarrow Y \times X
$$

to the point $\left(v_{0}, v_{0}\right)$ such that if $W_{k}^{\prime} \cap\left(v_{0} \times X\right) \neq \varnothing$, then $W_{k}^{\prime} \cap\left(v_{0} \times X\right)=U_{k}^{\prime}$ and $\left.T_{k}^{\prime}\right|_{U_{k}^{\prime} \times I}=H_{k}^{\prime}$, and if $W_{k}^{\prime} \cap\left(Y \times v_{0}\right) \neq \varnothing$, then $W_{k}^{\prime} \cap\left(Y \times v_{0}\right)=V_{k},\left.T_{k}^{\prime}\right|_{V_{k}^{\prime} \times I}=G_{k}^{\prime}$ for $k=0, \ldots, m+n$.

We define the open sets

$$
O_{k}=W_{k} \cup W_{k}^{\prime} \cup \tilde{U}_{k} \cup \tilde{V}_{k} \subset(X \vee Y) \times(X \vee Y), \quad k=0, \ldots, n+m,
$$

and note that $\mathcal{O}=\left\{O_{k}\right\}$ covers $(X \vee Y) \times(X \vee Y)$. Note that the set

$$
C=(X \vee Y) \times v_{0} \bigcup v_{0} \times(X \vee Y)
$$

defines a partition of $(X \vee Y) \times(X \vee Y)$ into four pieces: $X \times X, X \times Y, Y \times X$, and $Y \times Y$. Also note the inclusion $O_{k} \cap C \subset U_{k} \cup V_{k} \cup U_{k}^{\prime} \cup V_{k}^{\prime}$. By the construction the deformations $D_{X}^{k}, D_{Y}^{k}, T_{k}$, and $T_{k}^{\prime}$ all agree on $O_{k} \cap C$. Therefore the union of deformations

$$
T_{k} \cup T_{k}^{\prime} \cup D_{X}^{k} \cup D_{Y}^{k}: O_{k} \times I \rightarrow(X \vee Y) \times(X \vee Y)
$$

is a well-defined deformation $Q_{k}$ of $O_{k}$ to the diagonal $\Delta(X \vee Y)$. Note that for all $k, Q_{k}$ are strict deformations. By Proposition 3.1 each $Q_{k}$ defines a reserved section $\alpha_{k}: O_{k} \rightarrow P(X \vee Y)$. Therefore,

$$
\mathrm{TC}^{M}(X \vee Y) \leq n+m=\mathrm{TC}^{M}(X)+\mathrm{TC}^{M}(Y) .
$$

Remark 3.7. A stronger version of the upper bound of Theorem 3.6 was proposed in [F2], Theorem 19.1:

$$
\mathrm{TC}(X \vee Y) \leq \max \left\{\mathrm{TC}(X), \mathrm{TC}(Y), \operatorname{cat}_{\mathrm{LS}}(X)+\operatorname{cat}_{\mathrm{LS}}(Y)\right\}
$$

Since the proof in [F2 is incomplete, we call this inequality Farber's Conjecture. Note that Farber's inequality in view of Theorem 3.6 would turn into an equality for spaces $X$ and $Y$ satisfying the product law: $\operatorname{cat}_{\mathrm{LS}}(X \times Y)=\operatorname{cat}_{\mathrm{LS}}(X)+\operatorname{cat}_{\mathrm{LS}}(Y)$.

Theorem 3.8. (1) There is a 2-to-1 covering map $p: E \rightarrow B$ with $\mathrm{TC}(E)>$ $\mathrm{TC}(B)$.

(2) There is a finite complex $X$ with $\mathrm{TC}(X)<\mathrm{TC}(\tilde{X})$, where $\tilde{X}$ is the universal covering of $X$. 
Proof. (1) We take $B=T \vee S^{1}$, where $T=S^{1} \times S^{1}$ is a 2-torus. Let $E$ be the covering space defined by the 2 -fold covering of $S^{1}$. Note that $E$ is homeomorphic to the circle with two tori $T$ attached at antipodal points. Thus, $E$ is homotopy equivalent to $T \vee T \vee S^{1}$. By Theorem 3.6 and Lemma 2.7

$$
\mathrm{TC}(B) \leq \mathrm{TC}^{M}(T)+\mathrm{TC}^{M}\left(S^{1}\right)=\operatorname{cat}_{\mathrm{LS}}(T)+\operatorname{cat}_{\mathrm{LS}}\left(S^{1}\right)=2+1=3 .
$$

On the other hand, by Theorem 3.6 .

$$
\left.\mathrm{TC}(E) \geq \operatorname{cat}_{\mathrm{LS}}\left(\left(T \vee S^{1}\right) \times T\right)\right) \geq \operatorname{cup}-\text { length }\left(\left(T \vee S^{1}\right) \times T\right)=4 .
$$

(2) Consider $X=\left(S^{3} \times S^{3}\right) \vee S^{1}$. Since $S^{3} \times S^{3}$ is a connected Lie group, by Lemma 2.7. $\mathrm{TC}^{M}\left(S^{3} \times S^{3}\right)=\operatorname{cat}_{\mathrm{LS}}\left(S^{3} \times S^{3}\right)=3$. By Theorem 3.6 and Lemma 2.7. $\mathrm{TC}(X) \leq \mathrm{TC}^{M}\left(S^{3} \times S^{3}\right)+\mathrm{TC}^{M}\left(S^{1}\right)=\operatorname{cat}_{\mathrm{LS}}\left(S^{3} \times S^{3}\right)+\operatorname{cat}_{\mathrm{LS}}\left(S^{1}\right)=2+1=3$.

Note that the universal cover $\tilde{X}$ is homotopy equivalent to an infinite wedge

$$
Y=\bigvee^{\infty}\left(S^{3} \times S^{3}\right)
$$

Then $Y$ admits a retraction onto $\left(S^{3} \times S^{3}\right) \vee\left(S^{3} \times S^{3}\right)$. By Lemma 3.2, Theorem 3.6, and the cup-length lower bound on cat ${ }_{\mathrm{LS}}$,

$$
\mathrm{TC}(\tilde{X}) \geq \mathrm{TC}\left(\left(S^{3} \times S^{3}\right) \vee\left(S^{3} \times S^{3}\right)\right) \geq \operatorname{cat}_{\mathrm{LS}}\left(S^{3} \times S^{3} \times S^{3} \times S^{3}\right) \geq 4 .
$$

\section{Topological complexity, LS-Category, and Schwarz genus}

We say a subset $A \subset X$ can be rel $\infty$ contracted to infinity if for every compact subset $F \subset X$ there is a larger compact set $F \subset C$ and a homotopy $h_{t}: A \rightarrow X$ with $h_{0}=\operatorname{incl}_{A} \subset X, h_{1}(A) \cap F=\varnothing$, and $h_{t}(a)=a$ for $a \in A \backslash C$.

Definition 4.1. We define the normalized $r e l \infty$ category $\infty-\operatorname{cat}_{\mathrm{LS}}(X)$ of a locally compact space $X$ as the minimal $k$ such that there is a cover $X=V_{0} \cup V_{1} \cup \cdots \cup V_{k}$ by $k+1$ closed subsets where each $V_{i}$ can be rel $\infty$ contracted to infinity.

It follows from the definition that for every locally compact space $X$, $\operatorname{cat}_{\mathrm{LS}}(\alpha X)$ $\leq \infty$ - $\operatorname{cat}_{\mathrm{LS}}(X)$, where $\alpha X$ is the one-point compactification of $X$.

Question 4.2. Does the equality $\operatorname{cat}_{\mathrm{LS}}(\alpha X)=\infty$ - $\operatorname{cat}_{\mathrm{LS}}(X)$ hold for all locally finite complexes with tame ends?

We recall that $X$ has a tame end if there is a compactum $C \subset X$ such that $X \backslash \operatorname{Int}(C)$ is homeomorphic to $\partial C \times[0,1)$.

Since the one-point compactification of $X \times X$ with the diagonal $\Delta X$ removed is the quotient space $(X \times X) / \Delta X$, the following theorem shows that Question 4.2 is closely related to characterization of the topological complexity $\mathrm{TC}^{M}$ by means of the LS-category.

Theorem 4.3. For any compact ENR $X$,

$$
\operatorname{cat}_{\mathrm{LS}}(X \times X) / \Delta X \leq \mathrm{TC}^{M}(X) \leq \infty-\operatorname{cat}_{\mathrm{LS}}((X \times X) \backslash \Delta X) .
$$

Proof. The proof of the first inequality was presented informally in GV2 after Corollary 9. Suppose that $\mathrm{TC}^{M}(X)=k$. Then by the definition there is an open cover $U_{0}, U_{1}, \ldots, U_{k}$ of $X \times X$ with continuous reserved sections $s_{i}: U_{i} \rightarrow P X$ of $\pi: P X \rightarrow X \times X$. By Proposition 3.1 there are strict deformations of $U_{i}$ in $X \times X$ to the diagonal $\Delta X$. They define the deformations of $U_{i} /\left(U_{i} \cap \Delta X\right)$ to the point $\{\Delta X\}$ in $(X \times X) / \Delta X$. Thus, $\operatorname{cat}_{\mathrm{LS}}(X \times X) / \Delta X \leq k$. 
Let $\infty$ - $\operatorname{cat}_{\mathrm{LS}}((X \times X) \backslash \Delta X)=k$ and let $(X \times X) \backslash \Delta X=F_{0} \cup F_{1} \cup \cdots \cup F_{k}$ be the union of $k+1$ closed sets rel $\infty$ contractible to infinity. Let $W$ be a neighborhood of the diagonal $\Delta X$ in $X \times X$ that admits a deformation retraction $r_{t}$ to $\Delta X$. Let $h_{t}^{i}$ be a deformation of $F_{i}$ into $W$. Then the concatenation of $h_{t}^{i}$ and $r_{t}$ defines a deformation $H_{i}$ of $F_{i}$ to the diagonal. Let $\bar{F}_{i}=F_{i} \cup \Delta X$. Note that $H_{i}$ together with identity on $\Delta X$ defines a strict deformation of $\bar{F}_{i}$ to the diagonal.

Theorem 4.3 together with Theorem 2.5 recover Theorem 10(2) of GV2]:

Corollary 4.4. For a $k$-connected $C W$ complex $X$,

$$
\operatorname{cat}_{\mathrm{LS}}((X \times X) / \Delta X) \leq \mathrm{TC}(X),
$$

provided $(k+1)(\mathrm{TC}(X)+1)>\operatorname{dim} X+1$.

Proof. By Theorem 2.5, $\mathrm{TC}(X)=\mathrm{TC}^{M}(X)$ in this case.

The relations between $\mathrm{TC}(X), T C^{M}(X)$, and $\operatorname{cat}_{\mathrm{LS}}(X \times X) / \Delta X$ are still unknown. It could be that all three invariants coincide. A combination of results of GV2] and this paper shows that they do coincide for large classes of spaces. In view of Theorem 4.3 we believe the following might be true:

Conjecture 4.5. For $C W$ complexes $X$, $\mathrm{TC}^{M}(X)=\operatorname{cat}_{\mathrm{LS}}(X \times X) / \Delta X$.

It seems rather surprising that Theorem 10(2) of [GV2] and Theorem 2.5 have the same conditions. Perhaps this is another indication in favour of this conjecture.

Remark 4.6. For the topological complexity $\mathrm{TC}(X)$ a weaker version of the first inequality from Theorem 4.3 was proven in [F2], Lemma 18.3:

$$
\operatorname{cat}_{\mathrm{LS}}(X \times X) / \Delta X-1 \leq \mathrm{TC}(X) .
$$

Thus Theorem 4.3 suggests an approach to a possible counterexample to the Iwase-Sakai conjecture. Namely, if one finds a space $X$ where the above Farber's inequality is sharp, by Theorem 4.3 we would obtain $\mathrm{TC}^{M}(X)>\operatorname{TC}(X)$. In view of Theorem 2.5 such space in the non-simply connected case should satisfy the inequality $\mathrm{TC}(X) \leq \operatorname{dim} X$. The computation of the topological complexity of real projective spaces $([\mathrm{FTY}])$ produces an example of such $X=\mathbb{R} P^{3}$. But $\mathbb{R} P^{3}$ cannot be a counterexample to the Iwase-Sakai conjecture, since it is a Lie group (see Lemma 2.7). Moreover, projective spaces could not give a counterexample to the Iwase-Sakai conjecture in this approach in view of the equality $\mathrm{TC}\left(\mathbb{R} P^{n}\right)=\operatorname{cat}_{\mathrm{LS}}\left(\left(\mathbb{R} P^{n} \times \mathbb{R} P^{n}\right) / \Delta \mathbb{R} P^{n}\right)$ proven for all $n$ in GV2, Theorem 11. Perhaps the simplest space for which it's unknown whether $\mathrm{TC}=\mathrm{TC}^{M}$ is the Heisenberg manifold $H^{3}$. We recall that $H^{3}$ can be viewed as the pull-back of the Hopf bundle $S^{3} \rightarrow S^{2}$ by means of a degree one map $f: S^{1} \times S^{1} \rightarrow S^{2}$.

Question 4.7. What is $\operatorname{cat}_{\mathrm{LS}}\left(H^{3} \times H^{3}\right) / \Delta H^{3}$ ?

As was mentioned, the topological complexity of $X$ equals the Schwarz genus of a certain fibration. It turns out that for general fibrations we still have the inequalities similar to Theorem 4.3 .

Theorem 4.8. For any fibration of compact spaces $p: X \rightarrow Y$,

$$
\operatorname{cat}_{\mathrm{LS}}\left(C_{p}\right)-1 \leq s g(p) \leq \infty-\operatorname{cat}_{\mathrm{LS}}\left(C_{p} \backslash\{*\}\right) .
$$


Proof. We claim that if a subset $U \subset Y$ admits a section $s: U \rightarrow X$, then $U$ is contractible in $C_{p}$. Indeed, it can be moved to $X$ in the mapping cylinder $M_{p}$. Since the cone $\operatorname{Con}(X)$ is contained in $C_{p}$, it could be further contracted to a point. Moreover, the mapping cylinder $\hat{U}=M_{\left.p\right|_{p^{-1}(U)}}$ of the restriction of $p$ to the preimage $p^{-1}(U)$ is contractible in $C_{p}$, since it can be pushed to $U$ first. If $Y$ is covered by $n+1$ open sets $U_{0}, \ldots, U_{n}$, each of which admits a section of $p$, then the mapping cylinder $M_{p}$ can be covered by $n+1$ sets $\hat{U}_{0}, \ldots, \hat{U}_{n}$, all contractible in the mapping cylinder $C_{p}$. Since $C_{p}=M_{p} \cup \operatorname{Con}(X)$, the open enlargements of the sets $\hat{U}_{0}, \ldots, \hat{U}_{n}$, and $\operatorname{Con}(X)$ define an open cover of $C_{p}$ by $n+1$ elements, all contractible in $C_{p}$. Hence $\operatorname{cat}_{\mathrm{LS}}\left(C_{p}\right)-1 \leq s g(p)$.

Suppose that $\infty$ - $\operatorname{cat}_{\mathrm{LS}}\left(C_{p} \backslash\{*\}\right) \leq n$. Let $V_{0}, \ldots, V_{n}$ be a closed cover of $C_{p} \backslash\{*\}$ by sets that can be rel $\infty$ contracted to infinity. Let

$$
H_{i}: V_{i} \times I \rightarrow C_{p} \backslash\{*\}
$$

be a contraction such that

$$
H_{i}\left(V_{i} \times 1\right) \subset \operatorname{Con}(X) \backslash\{*\} \subset C_{p} \backslash\{*\} .
$$

We define $F_{i}=V_{i} \cap Y \subset C_{p}$. Let $\pi: \operatorname{Con}(X) \backslash\{*\} \rightarrow X$ be the projection. By the Homotopy Lifting Property, the homotopy $\left.p \circ H_{i}\right|_{F_{i} \times[0,1]}: F_{i} \times[0,1] \rightarrow Y$ has a lift $H_{i}^{\prime}: F_{i} \times[0,1] \rightarrow X$ which coincides with $\pi \circ H_{i}$ on $F_{i} \times 1$. Then $H_{i}^{\prime}$ restricted to $F_{i} \times 0$ is a section of $p$ over $F_{i}$. Thus, $s g(p) \leq \infty-\operatorname{cat}_{\mathrm{LS}}\left(C_{p} \backslash\{*\}\right)$.

The following example shows that neither of the two inequalities of Theorem 4.8 can be improved.

Example 4.9. (1) For the identity map $1_{X}: X \rightarrow X$ in view of the equality $C_{1_{X}}=\operatorname{Con}(X)$ we obtain

$$
\operatorname{cat}_{\mathrm{LS}}\left(C_{1_{X}}\right)-1=-1<s g\left(1_{X}\right)=0=\operatorname{cat}_{\mathrm{LS}}(\operatorname{Con}(X))=\infty-\operatorname{cat}_{\mathrm{LS}}\left(C_{1_{X}} \backslash\{*\}\right) .
$$

For the square map $p: S^{1} \rightarrow S^{1}, p(z)=z^{2}$,

$$
\operatorname{cat}_{\mathrm{LS}}\left(C_{p}\right)-1=1=s g(p)<2=\operatorname{cat}_{\mathrm{LS}}\left(C_{p}\right) \leq \infty-\operatorname{cat}_{\mathrm{LS}}\left(C_{p} \backslash\{*\}\right),
$$

since $C_{p}=\mathbb{R} P^{2}$ and cat $_{\mathrm{LS}}\left(\mathbb{R} P^{2}\right)=2$.

\section{ACKNOWLEDGEMENTS}

The author is thankful to Michael Farber and Yuli Rudyak for helpful conversations and to Peter Landweber for valuable remarks. Also, the author would like to thank the referee for acquainting him with the recent work on the subject by Garcia Calcines and Vandembroucq GV1], GV2].

\section{REFERENCES}

[BH] I. Berstein and P. J. Hilton, Category and generalized Hopf invariants, Illinois J. Math. 4 (1960), 437-451. MR0126276 (23 \#A3572)

[CLOT] Octav Cornea, Gregory Lupton, John Oprea, and Daniel Tanré, Lusternik-Schnirelmann category, Mathematical Surveys and Monographs, vol. 103, American Mathematical Society, Providence, RI, 2003. MR1990857 (2004e:55001)

[Dr] Alexander Dranishnikov, The Lusternik-Schnirelmann category and the fundamental group, Algebr. Geom. Topol. 10 (2010), no. 2, 917-924, DOI 10.2140/agt.2010.10.917. MR2629770 (2011h:55007)

[F1] Michael Farber, Topological complexity of motion planning, Discrete Comput. Geom. 29 (2003), no. 2, 211-221, DOI 10.1007/s00454-002-0760-9. MR1957228(2004c:68132) 
[F2] Michael Farber, Topology of robot motion planning, Morse theoretic methods in nonlinear analysis and in symplectic topology, NATO Sci. Ser. II Math. Phys. Chem., vol. 217, Springer, Dordrecht, 2006, pp. 185-230, DOI 10.1007/1-4020-4266-3_05. MR2276952 (2008d:68141)

[F3] Michael Farber, Instabilities of robot motion, Topology Appl. 140 (2004), no. 2-3, 245266, DOI 10.1016/j.topol.2003.07.011. MR2074919 (2005g:68166)

[F4] Michael Farber, Invitation to topological robotics, Zurich Lectures in Advanced Mathematics, European Mathematical Society (EMS), Zürich, 2008. MR2455573(2010a:55018)

[FTY] Michael Farber, Serge Tabachnikov, and Sergey Yuzvinsky, Topological robotics: motion planning in projective spaces, Int. Math. Res. Not. 34 (2003), 1853-1870, DOI 10.1155/S1073792803210035. MR1988783 (2004i:55005)

[GV1] J. Garcia-Calcines and L. Vandembroucq, Weak sectional category, J. Lond. Math. Soc. (2) 82 (2010), no. 3, 621-642, DOI 10.1112/jlms/jdq048. MR2739059 (2012b:55005)

[GV2] J. M. Garcia Calcines and L. Vandembroucq, Topological complexity of the homotopy cofibre of the diagonal map, Math. Z. 274 (2013), no. 1-2, 145-165. MR.3054321

[IS1] Norio Iwase and Michihiro Sakai, Topological complexity is a fibrewise L-S category, Topology Appl. 157 (2010), no. 1, 10-21, DOI 10.1016/j.topol.2009.04.056. MR2556074 (2011e:55009)

[IS2] Norio Iwase and Michihiro Sakai, Erratum to "Topological complexity is a fibrewise LS category" [Topology Appl. 157 (1) (2010) 10-21] [MR2556074], Topology Appl. 159 (2012), no. 10-11, 2810-2813, DOI 10.1016/j.topol.2012.03.009. MR2923451

[J] I. M. James, On category, in the sense of Lusternik-Schnirelmann, Topology 17 (1978), no. 4, 331-348, DOI 10.1016/0040-9383(78)90002-2. MR.516214 (80i:55001)

[Os] Phillip A. Ostrand, Dimension of metric spaces and Hilbert's problem 13, Bull. Amer. Math. Soc. 71 (1965), 619-622. MR0177391 (31 \#1654)

[Sch] A. S. Schwarz, The genus of a fiber space, Amer. Math. Soc. Transl. (2), 55, Amer. Math. Soc., Providence, RI, 1966, pp. 49-140.

[TD] Tammo tom Dieck, Algebraic topology, EMS Textbooks in Mathematics, European Mathematical Society (EMS), Zürich, 2008. MR2456045 (2009f:55001)

Department of Mathematics, 358 Little Hall, University of Florida, Gainesville, FLORIDA 32611-8105

E-mail address: dranish@math.ufl.edu 